\title{
Identification of Stress Corrosion Cracking Profiles Using \&-Greedy Search Inverse Analysis in Eddy Current Testing
}

\author{
Fumio Kojima ${ }^{1}$, Teruo Usami ${ }^{2}$, and Nguyen Thanh Duong ${ }^{1}$ \\ 1 Graduate School of Science and Technology, Kobe University, Kobe, Japan \\ (Phone/Fax +81-78-803-6493; E-mail: koima(okoala.kobe-4a.ac.3p) \\ 2 Advanced Technology R \& D Center, Mitsubishi Electric Co., Amagasaki, Japan \\ (E-mail: Usami Teruo@as Misubibhelectric co jo)
}

\begin{abstract}
This paper is concerned with a method to recover natural cracks from magnetic images obtained by ECT (eddy current testing). In the proposed method, a Database is built to store knowledge base of cracks with different sizes and various measurement parameters. In the proposed Inverse Analysis, $\varepsilon$-Greedy search algorithm invokes knowledge from pre-built database to reconstruct multiple cracks from ECT signals.
\end{abstract}

\section{Introduction}

Recently, quantitative nondestructive evaluation (QNDE) has played an important role in structural integrity of material system used in nuclear power plants. To this end, eddy current testing technology has been widely used for QNDE.

Many advance scanning methods have been developed in ECT technology. The most recently developed ECT sensors composed of arrayed-coil type ECT probes have drastically improved efficiency of inspection in terms of scanning speed and detect ability in the surface.

In the sensors, pairs of coil, transmitter and receiver coils are arranged in arrays as shown in Fig. 1. There are two scanning patterns used in this scanning technique: T-Scan and U-Scan. ECT signals measured by these two patterns are both used in Inverse Analysis for recovery cracks, as will be discussed here after.

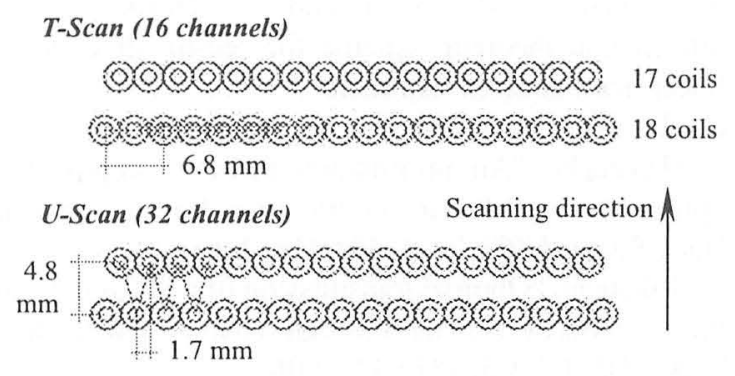

Fig. 1 Scanning strategies of arrayed type ECT probes

The proposed method for recovering multiple cracks is composed of two parts. Firstly, a Database is built, using ECT simulation and measurement results of electric discharge machining (EDM) slits in SUS316L sample. Secondly, Inverse Analysis invokes knowledge base of cracks with different size and certain scanning parameters from database to reconstruct combination of cracks from scanned ECT signal.

This paper is organized as follows: In section 2, strategy to build knowledge base of cracks, called ECT Database, is discussed. In section 3, an inverse analysis with $\varepsilon$-Greedy search algorithm is proposed for reconstructing crack profiles from ECT signals obtained by multiple coil probes. Partial comparison to developed Greedy search and SSGA method will be shown.

\section{ECT Database}

Database is crucial part of this work. This database stores knowledge about ECT signals of cracks with different sizes and various scanning parameters, such as driving frequency, scanning patterns and scanning positions (assuming that all crack is rectangular and perpendicular to scanning direction).

In building ECT database, ECT simulation with Nodal-based FEM model derived from Maxwell's equations (the so-called A- $\varphi$ model) is used. Decomposition of ECT simulation is shown in Fig. 2.
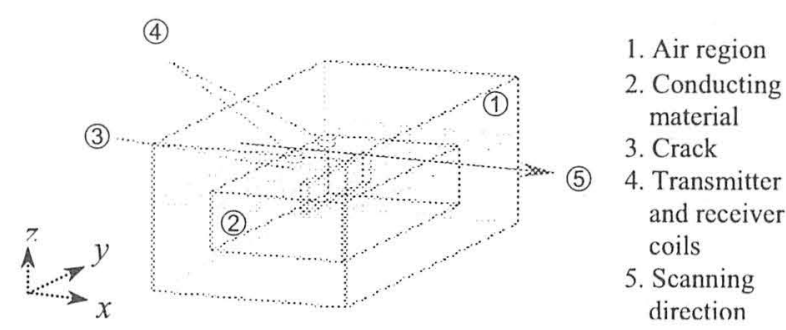

Fig. 2 Decomposition of ECT simulation model

Outputs of this ECT simulation are Simulation magnetic images (including real part and imaginary part of voltage) of EDM cracks. The size of Simulation magnetic images are $18.1 \mathrm{~mm}$ in $\mathrm{x}$-direction with 181 scan points ( $\mathrm{x}$-pitch is $0.1 \mathrm{~mm})$ and $54.4 \mathrm{~mm}$ in $\mathrm{y}$-direction with 16 scan lines (T-Scan) and 32 scan lines (U-Scan). 
Using this ECT simulation, magnetic images of EDM cracks are created. Table 1 and Fig. 3 depict description of cracks in ECT simulation.

Table 1 Crack description in ECT simulation

\begin{tabular}{|l|l|}
\hline Crack depth & $1,2,3,5,7,10 \mathrm{~mm}$ \\
\hline Crack length & $1,2, \ldots, 14 \mathrm{~mm}$ \\
\hline Crack width & $0.3 \mathrm{~mm}$ \\
\hline $\begin{array}{l}\text { Crack position } \\
\text { in x-direction }\end{array}$ & In the middle of the magnetic image \\
\hline $\begin{array}{l}\text { Crack position } \\
\text { in y-direction }\end{array}$ & $\begin{array}{l}-7 \mathrm{~mm} \text { to } 7 \mathrm{~mm} \\
\text { (to the fist scan line in y-direction) }\end{array}$ \\
\hline
\end{tabular}

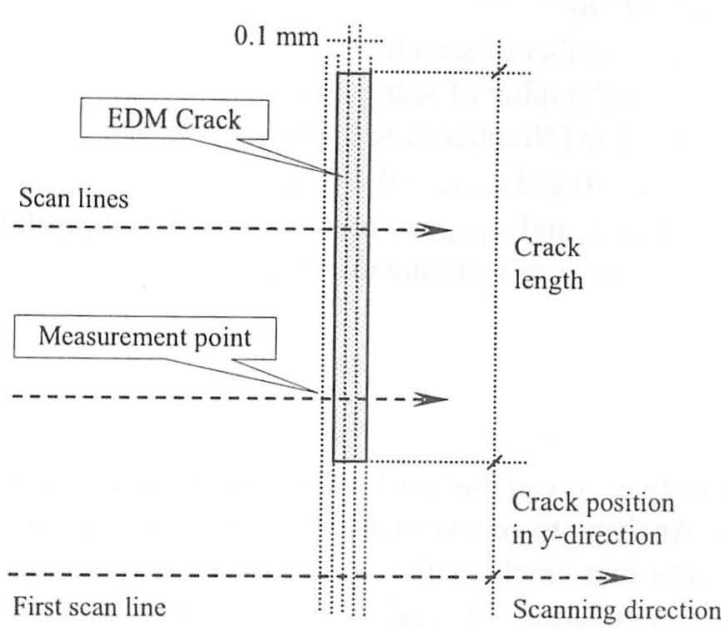

Fig. 3 Crack description in ECT simulation

In order to increase the accuracy of ECT simulation data, outputs of ECT simulation are verified and adjusted by measurement results of EDM slits in SUS316L sample.

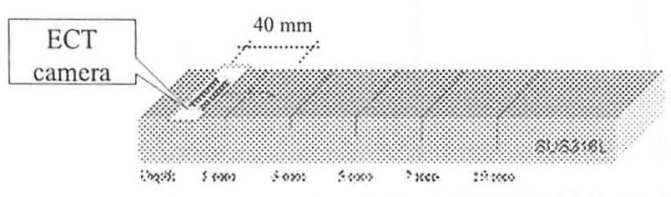

Fig. 4 SUS316L sample

Table 2 Details of EDM slits on SUS316L sample

\begin{tabular}{|c|c|c|c|c|c|}
\hline & EDM 1 & EDM 2 & EDM 3 & EDM 4 & EDM 5 \\
\hline $\begin{array}{c}\text { Length } \\
(\mathrm{mm})\end{array}$ & 150 & 150 & 150 & 150 & 150 \\
\hline $\begin{array}{c}\text { Depth } \\
(\mathrm{mm})\end{array}$ & 1 & 3 & 5 & 7 & 10 \\
\hline $\begin{array}{c}\text { Width } \\
(\mathrm{mm})\end{array}$ & 0.3 & 0.3 & 0.3 & 0.3 & 0.3 \\
\hline Shape & $20 \mathrm{MHz}, 40 \mathrm{MHz}$ and $100 \mathrm{MHz}$ \\
\hline $\begin{array}{c}\text { Driving } \\
\text { frequency }\end{array}$ & \multicolumn{5}{|c|}{ Rectangle } \\
\hline
\end{tabular}

Output of ECT simulation is able to be classified by: driving frequency, scanning pattern (T-Scan or U-Scan), crack depth, crack length and crack position in y-direction (or scanning direction). With the same driving frequency, scanning pattern, crack depth, simulation data vary in different crack length and scan line (position in $\mathrm{y}$-direction).

In case that crack length equals $14 \mathrm{~mm}$ and scan line is in the middle of crack, simulation data should be exactly the same with measurement data form SUS316L sample. Because the length of all slits in SUS316L sample is 150 $\mathrm{mm}$, all scan line data of the sample can be supposed to be in the middle of crack.

Derived from this remarks, the adjustment rules is set up as follows:

\section{Algorithm:}

- With simulation data of the same driving frequency, scanning pattern and crack depth:

Step 1: Select simulation data of scan line in the middle of crack with crack length equals $14 \mathrm{~mm}$.

Step 2: Compare simulation data from Step 1 with measurement data from SUS316L sample (with compatible driving frequency, scanning pattern and crack depth). Than, set up conversion rule to adjust simulation data.

After applying this conversion rule to simulation data of the scan line selected in Step 1, simulation data must be exactly the same compared with measurement data from SUS316L sample.

Step 3: Apply conversion rule created in Step 2 to simulation data of every scan line on different crack length but the same driving frequency, scanning pattern and crack depth.

- Repeat these 3 steps with simulation data in different driving frequency, scanning pattern and crack depth. End.

After adjusting simulation outputs with measurement results of SUS316L, data is stored in MySQL database for later use in Inverse Analysis. Table 3 and Table 4 show structure of data being stored in ECT database.

Table 3 Structure of Crack table

\begin{tabular}{|l|l|l|l|}
\hline $\begin{array}{l}\text { Column } \\
\text { name }\end{array}$ & Description & $\begin{array}{l}\text { Data } \\
\text { type }\end{array}$ & Comments \\
\hline CrackID & Crack identification & Numeric & Key \\
\hline Depth & Crack depth & Integer & x $0.1 \mathrm{~mm}$ \\
\hline Length & Crack length & Integer & x $0.1 \mathrm{~mm}$ \\
\hline Width & Crack width & Double & $0.3 \mathrm{~mm}$ \\
\hline
\end{tabular}

Table 4 Structure of Risaj table

\begin{tabular}{|l|l|l|l|}
\hline $\begin{array}{l}\text { Column } \\
\text { name }\end{array}$ & Description & $\begin{array}{l}\text { Data } \\
\text { type }\end{array}$ & Comments \\
\hline CrackID & Crack identification & Numeric & Foreign Key \\
\hline Pattern & T-Scan or U-Scan & Binary & \\
\hline Frequency & 20,40 or $100 \mathrm{KHz}$ & Integer & \\
\hline Scan_Point & x-axis & Integer & x $0.1 \mathrm{~mm}$ \\
\hline Scan_Line & y-axis: Channel & Integer & x $0.1 \mathrm{~mm}$ \\
\hline Real & Real part of voltage & Double & volt \\
\hline Image & Imaginary part & Double & volt \\
\hline
\end{tabular}




\section{Inverse Analysis}

Inverse Analysis completes in three steps. In the first step, Pre-process examines measured ECT signals to estimate possible length and position of cracks (assuming that all cracks are perpendicular to scanning direction) and possible crack depth. Secondly, $\varepsilon$-Greedy search algorithm invokes ECT database to estimate the size and exact position of cracks, from which simulated magnetic image has minimum error in comparison with observation image obtained by ECT camera. Finally, Post-process examines observation ECT signals once again to estimate the shape of the bottom of each crack.

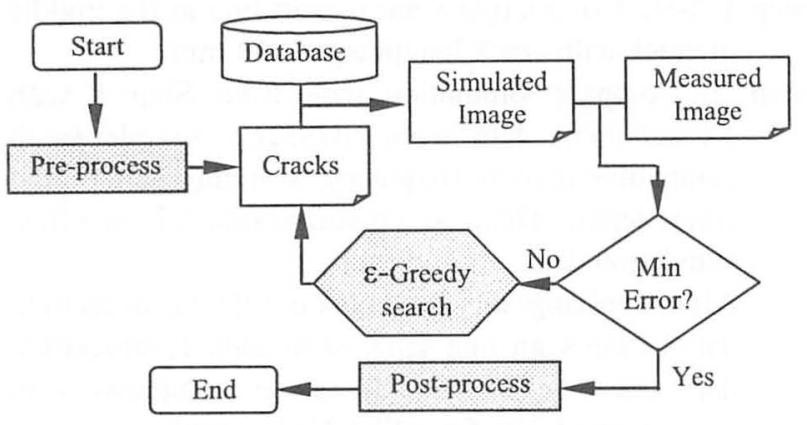

Fig. 5 Crack shape recovery flow chat

\subsection{Pre-process}

Pre-process module examines measured T-Scan signals to determine crack length and position; examines measured U-Scan signals to determine possible crack depth. After this step, a set of crack with initial size and position is created to be the input for the next step.

\subsubsection{Determine possible crack length and position}

In T-Scan pattern, the direction of transmitter and receiver coils is parallel to crack direction, as shown in Fig. 6. That make T-Scan signal is symmetric to the position of crack with the peak value at the middle of the crack in $\mathrm{x}$-axis.

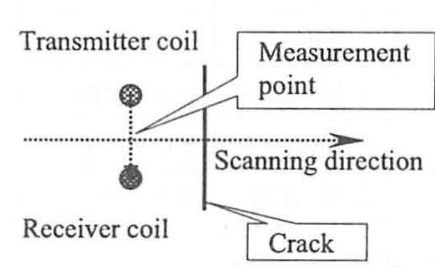

Fig. 6a

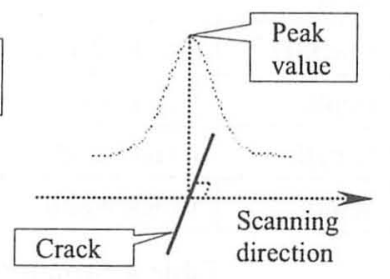

Fig. $6 b$
Fig. 6 (a) Decomposition of T-Scan pattern, (b) Characteristic of T-Scan signal.

Algorithm: (3.1a)

Step 1: Determine the possible position of $\operatorname{crack}(\mathrm{s})$ in each scan line of imaginary part of T-Scan signal (16 lines).

Step 2: Merge cracks determined in Step 1 to determine set of crack with possible length and position.

End.

After Step 1, a matrix $\left\{\mathrm{a}_{\mathrm{ij}}\right\}, \mathrm{a}_{\mathrm{ij}} \in\{0,1\}$ is created.
If $a_{i j}>0$, there is possible crack in scan line $i$ at position $\mathrm{j}$ (in $\mathrm{x}$-axis).

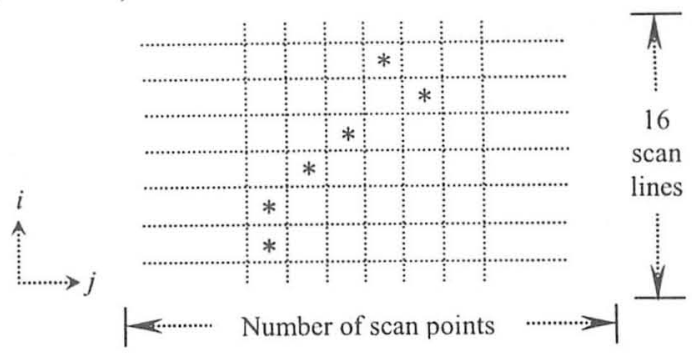

Fig. 7 Example of matrix $\left\{a_{i j}\right\}$ created by Step 1

In Step 2, possible crack is determined as follows: Algorithm: (3.1b)

For $i=1$ to [Number of scan lines]

For $j=1$ to [Number of scan points] \{

For $\mathrm{k}=1$ to [Number of scan points]

If $\mathrm{a}_{\mathrm{ij}}>0$ and $\mathrm{a}_{(\mathrm{i}+1) \mathrm{k}}>0$ and $|\mathrm{k}-\mathrm{j}|<\sigma$

Then $\mathrm{a}_{\mathrm{ij}}$ and $\mathrm{a}_{(\mathrm{i}+1) \mathrm{k}}$ are in the same crack at position $(k+j) / 2$ in scanning direction

\}

\}

\}

End.

In practice, $\sigma$ can be used as an input parameter for Inverse Analysis to recover natural crack, as illustrated in Fig.8a into one crack, with $\varepsilon$ is large enough (Fig.8b) or into a combination of cracks, with small $\sigma$ (Fig.8c), according to user's requirement.

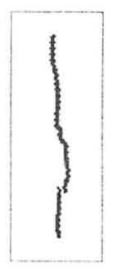

Fig. 8 a

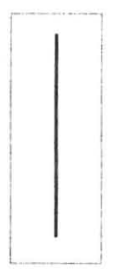

Fig. $8 \mathrm{~b}$

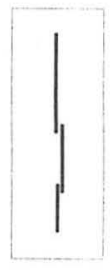

Fig. 8c
Fig. 8 Different result of crack recovery, depend on input parameter $\sigma$

After process (3.1.1), a set of crack with possible size and position is created to be the input for the next process (3.1.2).

\subsubsection{Determine possible crack depth}

Measurement results of EDM slits in SUS316L sample show: Peak values of imaginary part of U-Scan signal are noticeably different in different crack depth, as illustrated in Fig. 9.

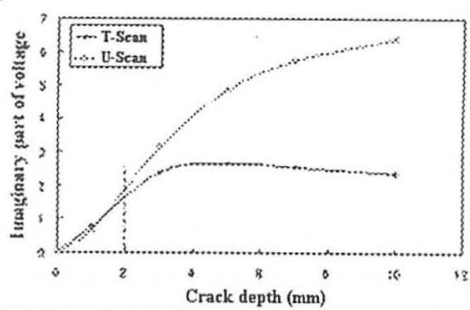

Fig. 9 Peak value of imaginary part of voltage (U-Scan)

The point at this process is finding peak value of imaginary part of voltage in U-Scan measured signal and 
compare with measurement results of EDM slits in SUS316L sample to determine possible depth of each crack created from process described in 3.1.1.

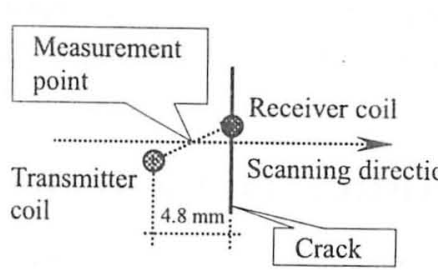

Fig. 10a

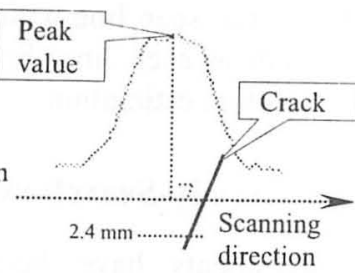

Fig. 10b
Fig. 10 (a) Decomposition of U-Scan pattern,

(b) Characteristic of U-Scan signal

As shown in Fig. 10, peak value of imaginary part of voltage in U-Scan measured signal can be located at 2.4 $\mathrm{mm}$ before crack position in $\mathrm{x}$-axis. The algorithm to determine possible crack depth is given as follows:

Algorithm: (3.1c)

With each crack found from process (3.1.1) \{

- With each scan line across this crack \{

Pick the value of imaginary part from U-Scan signal at this scan line and $\mathrm{x}$-position at crack position - $2.4 \mathrm{~mm}$.

- Get the average peak value of all above scan lines.

- Compare this average value with measured data of SUS316L sample to determine possible crack depth.

$$
\text { \} }
$$

End.

In this part, characteristics of T-Scan and U-Scan signal have been review and proposed algorithm for Pre-process in Inverse Analysis has been shown. The output of Pre-process is a set of cracks with possible size and position, to be the input for the next process.

\section{2 \&-Greedy-Search Algorithm}

As shown in Fig. 5, $\varepsilon$-Greedy-Search process searches for the combination of cracks, in order to minimize the different between simulated magnetic image created by Inverse Analysis and measurement image obtained by ECT camera. The fitness is calculated by the following equation:

$$
E(C)=\sum_{x, y}\left(\left[\sum_{c \in C} S_{r}-M_{r}\right]^{2}+\left[\sum_{c \in C} S_{i}-M_{i}\right]^{2}\right)
$$

Where $\mathrm{x}, \mathrm{y}, \mathrm{C}, \mathrm{S}, \mathrm{M}, \mathrm{r}, \mathrm{i}$ are scan points, scan lines (or channels), set of crack, simulation data, measurement data, real part and imaginary part of voltage, respectively.

$\varepsilon$-Greedy-Search algorithm tries to find $\mathrm{E}^{*}=\min \mathrm{E}(\mathrm{C})$ in a certain range of position and size of cracks.

As discussed in 3.1 , by using certain information from T-Scan and U-Scan signal, Pre-process manages to init a set of crack with possible crack size and position from the measured magnetic image. Then, $\varepsilon$-Greedy-Search will search for each crack from this set in a certain range of length, depth and position to find the most appropriate value that minimize the fitness $\mathrm{E}^{*}=\mathrm{E}(\mathrm{C})$.

$\varepsilon$-Greedy-Search algorithm is proposed as follows:
Algorithm: (3.2)

Input:

- C: Original set of crack produced by Pre-process Cracks in this set is sorted by depth: deepest first

Each crack $c \in C$ is determined by $f_{c}(x, y, 1)$ where $x, y, 1$ are position in $\mathrm{x}$-axis, position in $\mathrm{y}$-axiz and crack length, respectively.

After Pre-process, each crack has been determined a initial state as $\mathrm{f}_{\mathrm{c}}\left(\mathrm{x}_{0}, \mathrm{y}_{0}, \mathrm{l}_{0}\right)$.

- Measured magnetic image.

- ECT Database (for producing simulated image)

- dx: Step search for cracks' position in $\mathrm{x}$-axis

- dy: Step search for cracks' position in y-axis

- dl: Step search for the length of cracks

- $E^{*}$ (Initialized fitness).

Output:

- $\mathrm{C}^{*}$ : Set of crack that make $\mathrm{E}^{*}=\min \mathrm{E}(\mathrm{C})$.

Begin

$\mathrm{C}^{*}=\mathrm{C}$

For each crack $\mathrm{c}$ in $\mathrm{C}\{$

For (crack depth $+/-1$ level) \{

e-Greedy(c) // Search for the best profile of crack c // to minimize $\mathrm{E}^{*}$

Reduce $d x, d y, d l(u n t i l d x=d y=d l=0)$.

\}

End.

Fig. 11 describes strategy to search for the most appropriate specification of one crack.

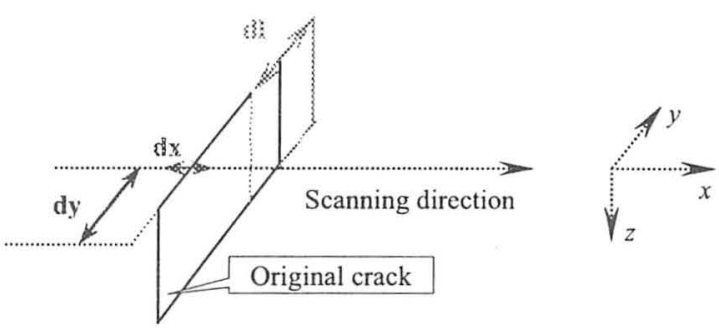

Fig. 11 Searching strategy

In order to calculate $E(C)$, Inverse Analysis invokes ECT Database to create simulation magnetic image and compare with measured image, using equation [1].

Detail of e-Greedy(c) function is given as follows: Algorithm: (3.3)

e-Greedy(c) $/ / \mathrm{c} \in \mathrm{C}$

Input:

Same as Algorithm: (3.2)

$\varepsilon$ (parameter)

Output:

$\mathrm{f}_{\mathrm{c}}\left(\mathrm{x}_{\mathrm{n}}, \mathrm{y}_{\mathrm{n}}, \mathrm{z}_{\mathrm{n}}\right)$

$\mathrm{E}_{\mathrm{c}} *=\min \mathrm{E}_{\mathrm{c}}(\mathrm{C})$

Begin

$\mathrm{i}=0$

While (not $\left.\mathrm{E}^{*}=\min \mathrm{E}(\mathrm{C})\right)$ \{

$\left\{E_{i}\right\}=\left\{E\left(C \mid f_{c}\left(x_{i}, y_{i}, 1_{i}\right)\right)\right\}$

$x_{i+1}=x_{i} \pm d x \quad / / x_{i+1}, y_{i+1}, l_{i+1}$ are selected in the same

$\mathrm{y}_{\mathrm{i}+1}=\mathrm{y}_{\mathrm{i}} \pm \mathrm{dy} \quad / /$ direction with $\mathrm{x}_{\mathrm{i}}, \mathrm{y}_{\mathrm{i}}, \mathrm{l}_{\mathrm{i}}$ compared

$\mathrm{l}_{\mathrm{i}+1}=\mathrm{l}_{\mathrm{i}} \pm \mathrm{dl} \quad$ // with initial position $\mathrm{x}_{0}, \mathrm{y}_{0}, \mathrm{l}_{0}$ 


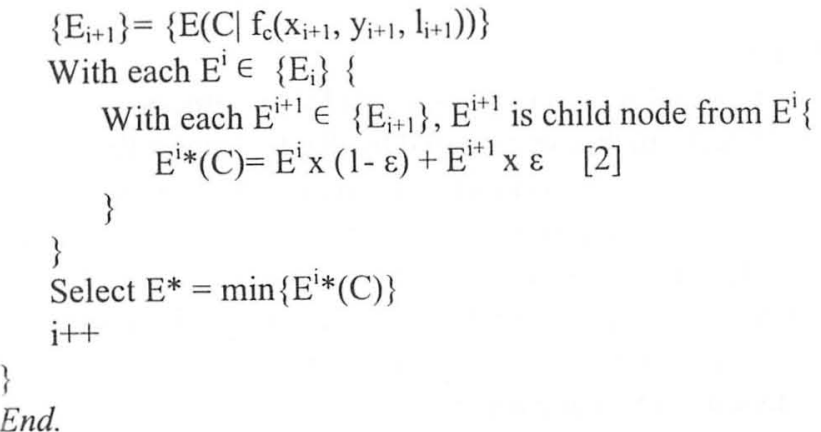

In this algorithm, each node in three-dimentional searching space is selected using fitness of this node and fitnesses of its children node, according to equation [2]. If $\varepsilon$ is set equal to zero, $\varepsilon$-Greedy-Search becomes Greedy-Search.

As a conclusion for this part, Fig. 12 illustrates the graph of fitness $E(C)$ and $E^{*}$ produced by $\varepsilon$-Greedy-Search algorithm.

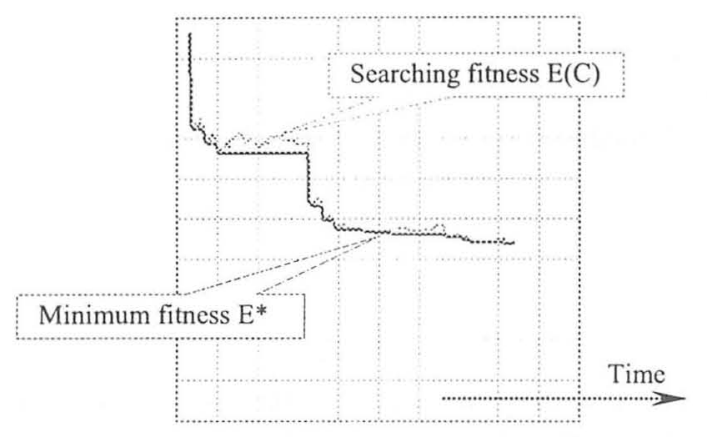

Fig. 12 Fitness in searching process

\subsection{Post-process}

$\varepsilon$-Greedy-Search algorithm has managed to produce a set of crack that closely matched measured magnetic image obtained by ECT scan. All recovered cracks are in rectangle shape with flat bottom. In practice, bottom of natural cracks are not in flat shape but deep in the middle and closed to the surface at the ends.

To estimate the bottom shape of recovered cracks, Post-process examines measured U-Scan signals to determine the peak of each scan line across the crack and compare with typical peak value of measured data of SUS316L sample to estimate the depth of each scan line; then draw the bottom of cracks.

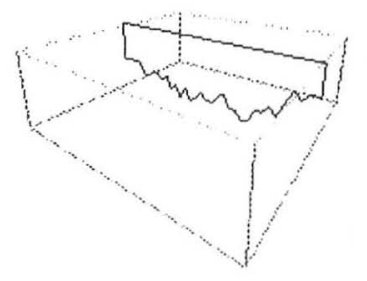

Fig. 13 Estimation the bottom of crack

Although measured data of SUS316L sample (shown in Fig. 9) have peak values of crack depth with 1, 2, 3, 5, 7, $10 \mathrm{~mm}$, peak values of crack depth $4,6,8,9 \mathrm{~mm}$ can be deducted from the graph in Fig. 9.
Having typical peak values of U-Scan signal in any crack depth, Post-process examines measured U-Scan signal of all scan lines across each crack and compare peak value of this scan line with typical peak values to estimate the depth in each line. Fig. 13 illustrates an example of crack bottom estimation.

\section{4 \&-Greedy-Search vs. Greedy Search and SSGA}

Experiments have been made to compare inverse analysis results among three methods: $\varepsilon$-Greedy-Search, Greedy-Search and SSGA. Table 5, 6 and Fig. 14 below shows an experiment system information and comparison on the inverse analysis results:

Table 5 Experiment system

\begin{tabular}{|l|l|}
\hline Computer system & $\begin{array}{l}\text { Intel Pentium } 4,3.4 \mathrm{GHz} \\
\text { 2GB RAM }\end{array}$ \\
\hline Operating system & Microsoft Windows 2000 \\
\hline $\begin{array}{l}\text { Programming language } \\
\text { Compiler }\end{array}$ & $\begin{array}{l}\mathrm{C} / \mathrm{C}++ \\
\text { Microsoft Visual C }++ \text { Express }\end{array}$ \\
\hline Interface & OpenGL \\
\hline
\end{tabular}

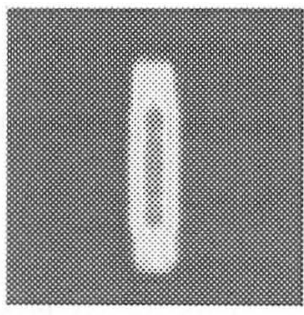

(1) Simulation. Noise free

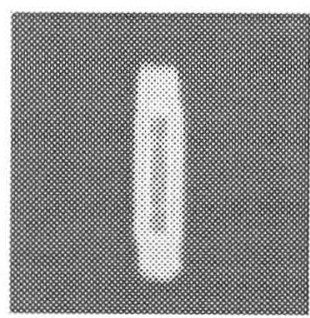

(3) Simulation. $\pm 25 \%$ noise

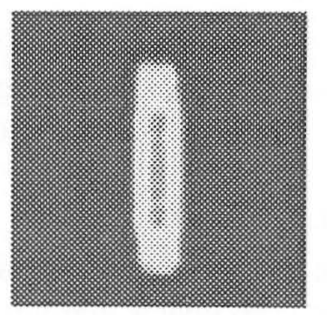

(2) Simulation. $\pm 10 \%$ noise

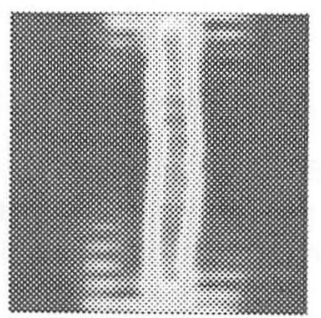

(4) Natural crack
Fig. 14 Magnetic images (U-Scan)

Table 6.1 Comparison between $\varepsilon$-Greedy-Search, Greedy-Search and SSGA (Natural crack)

\begin{tabular}{|c|c|c|c|c|c|}
\hline & \multirow[b]{2}{*}{$\varepsilon$} & \multicolumn{2}{|c|}{ One crack } & \multicolumn{2}{|c|}{ Three cracks } \\
\hline & & Fitness & Elapsed & Fitness & Elapsed \\
\hline \multirow{3}{*}{$\begin{array}{l}\text { OCusiy } \\
\text { Sowses: }\end{array}$} & 0.2 & $1.074 \mathrm{e}+04$ & \multirow{3}{*}{$21 \mathrm{~s}$} & $8.750 \mathrm{e}+03$ & \multirow{3}{*}{$63 \mathrm{~s}$} \\
\hline & 8.5 & $\$ .039604$ & & $8.7\{4: 3 \times 4\}$ & \\
\hline & 0.8 & $1.048 \mathrm{e}+04$ & & $8.794 e+03$ & \\
\hline \multicolumn{2}{|c|}{ Grocdy-Scarch } & $3044 \mathrm{e} \div 04$ & $13 \mathrm{~s}$ & 8.7700003 & $28 \mathrm{~s}$ \\
\hline \multicolumn{2}{|c|}{$\begin{array}{l}\text { SSGA ( } 50 \\
\text { genes, } 2000 \\
\text { generations) }\end{array}$} & $1.179 \mathrm{e}+04$ & $203 \mathrm{~s}$ & $9.682 \mathrm{e}+03$ & $462 \mathrm{~s}$ \\
\hline
\end{tabular}


Table 6.2 Comparison between $\varepsilon$-Greedy-Search, Greedy-Search and SSGA (Simulation crack)

\begin{tabular}{|c|c|c|c|c|}
\hline & $\varepsilon$ & Noise free & $\pm 10 \%$ noise & $\pm 20 \%$ noise \\
\hline \multirow{3}{*}{$\begin{array}{l}\varepsilon \text {-Greedy } \\
\text { Search }\end{array}$} & 0.2 & \multirow{5}{*}{$0.000 \mathrm{e}+00$} & \multirow{5}{*}{$5.969 \mathrm{e}+01$} & \multirow{5}{*}{$3.821 \mathrm{e}+02$} \\
\hline & 0.5 & & & \\
\hline & 0.8 & & & \\
\hline \multicolumn{2}{|c|}{ Greedy-Search } & & & \\
\hline \multicolumn{2}{|c|}{$\begin{array}{l}\text { SSGA ( } 50 \text { genes, } \\
2000 \text { generations) }\end{array}$} & & & \\
\hline
\end{tabular}

The comparison shows that $\varepsilon$-Greedy-Search, Greedy-Search are much faster than SSGA Inverse Analysis speed. Althought $\varepsilon$-Greedy-Search is not as fast as Greedy-Search, $\varepsilon$-Greedy-Search gives more accurate result in recovery natural crack profile (as shown in Table $6.1, \varepsilon=0.5)$.

\section{Conclusions}

We have developed electromagnetic inverse methodologies for sizing stress corrosion cracking for boiled water reactor (BWR) plants. It is very crucial to characterize target cracking of SUS304 and SUS316L materials used in BWR plants since those involve various kind of complexities, such as orientation, multiple deep-lying branching, partially conducting, etc. In this paper, we have proposed a model based health monitoring system for sizing natural cracking. The Greedy-Search inverse analysis and parallelization have managed to reduce the time consuming in inverse analysis process to make this method become reliable to be applied in ECT in-service inspections. The SCC sizing technique was applied to eddy current testing and experiments were effectively tested with laboratory data. The feasibility and validity of our algorithm using laboratory data are currently under study.

\section{References}

[1] F. Kojima, N. Kubota, F. Kobayashi and T. Takagi: Shape recovery of natural crack using evolutionary programming related to eddy current testing, International Journal of Applied Electromagnetic and Mechanics, Vol. 15, pp. 243-247 (2002)

[2] F. Kojima: Inverse Problems related to Electromagnetic Nondestructive Evaluation, Research Directions in Distributed Parameters System (R.C. Smith and M.A. Demetrious Eds.), Frontiers in Appl. Math. Vol. FR-27, pp. 219-236 (2003) SIAM

[3] F. Kojima and K. Ito: Reconstruction of magnetic charge densities using regularization of bounded variations under noisy observations, International Journal of Applied Electromagnetic and Mechanics, Vol. 19, pp. 309-313 (2004)

[4] F. Kojima, F. Kobayashi, F. Kawai, N. Kamezaki, S. Nishimizu, A. Koike, and M. Matsui: Development of hybrid nondestructive evaluation system using electromagnetic inverse analysis with measurements, Proceeding of SICE Annual Conference, Okayama, pp. 992-995 (2005) 